\title{
Algunas anotaciones sobre la importancia del cobre en la reproducción bovina
}

Gómez Rendón, Jorge; Del Campo, Marcelo; González Tous, Marco

Algunas anotaciones sobre la importancia del cobre en la reproducción bovina

Revista Colombiana de Ciencia Animal, vol. 11, núm. 1, 2019

Universidad de Sucre, Colombia

DOI: https://doi.org/10.24188/recia.v11.n1.2019.716

Los autores permiten a RECIA reimprimir el material publicado en él. En caso de que un autor quiera traducir o usar una publicación parcial o completa de nuestro Diario, el autor debe obtener un permiso por escrito del editor de la revista.

Esta obra está bajo una Licencia Creative Commons Atribución 4.0 Internacional. 


\section{Algunas anotaciones sobre la importancia del cobre en la reproducción bovina}

Some notes on the importance of copper in bovine reproduction

Jorge Gómez Rendón

DOI: https://doi.org/10.24188/recia.v11.n1.2019.716

Práctica privada, Colombia

jorgegomezmvz@hotmail.com

iD http://orcid.org/0000-0002-0719-7163

Recepción: 05 Noviembre 2018

Marcelo Del Campo

Aprobación: 18 Marzo 2019

Universidad Jorge Basadre, Facultad de Medicina

Publicación: 01 Abril 2019

Veterinaria y Zootecnia, Tacna, Perú., Perú

mrdelcampo@yahoo.es

iD http://orcid.org/0000-0001-9867-3884

Marco González Tous

Universidad de Córdoba. Facultad de Medicina

Veterinaria y Zootecnia. Departamento de Ciencias

Pecuarias. Instituto de Investigaciones Biológicas del Trópico

(IIBT). Montería, Colombia, Colombia

mgonzalezt@correo.unicordoba.edu.co

iD http://orcid.org/0000-0001-6500-5601

\section{Resumen:}

En esta breve revisión de tema se resumió información relacionada con algunas funciones del cobre en bovinos, sus requerimientos, deficiencias, toxicidad, efectos sobre la reproducción y algunos métodos de suplementación. También se abordó la importancia del cobre en la reproducción animal y algunas interrelaciones con otros minerales, síntomas generales de deficiencia y de intoxicación en bovinos. Igualmente se comentó los requerimientos nutricionales del cobre y la concentración concomitante del hierro, molibdeno y azufre. En conclusión se puede afirmar que un correcto balance mineral en la dieta animal, así como el aseguramiento de un adecuado consumo, supone una mejora en las tasas de preñez, tasa de natalidad, aumento de la producción en leche, mejores pesos al destete y ganancia de peso, mejorando la rentabilidad de los sistemas de producción bovina.

Palabras Clave: Aborto, azufre, cobre, minerales, molibdeno, reproducción (Fuente: DeCS).

\section{AbStraCt:}

In this brief review of the topic, information related to some functions of copper in cattle, their requirements, deficiencies, toxicity, effects on reproduction and some supplementation methods were summarized. The importance of copper in animal reproduction and some interrelations with other minerals, general symptoms of deficiency and intoxication in bovines were also addressed. The nutritional requirements of copper and the concomitant concentration of iron, molybdenum and sulfur were also discussed. In conclusion it can be said that a correct mineral balance in the animal diet, as well as the assurance of adequate consumption, implies an improvement in pregnancy rates, birth rate, increase in milk production, better weights at weaning and gain of weight, improving the profitability of bovine production systems.

KEYWORDS: Abortion, copper, minerals, molybdenum, sulfur, reproduction (Source: DeCS). 


\section{INTRODUCCIÓN}

En la mayoría de países de Latinoamérica las pasturas se constituyen en la principal fuente de alimento para los sistemas de producción bovina y Colombia no es la excepción en este aspecto; especialmente en los sistemas de producción extensivos; aunque no excluyente en otros sistemas. Por lo tanto se debe aprovechar el máximo de nutrientes contenidos en ellos con el fin de obtener gran parte de los requerimientos de los bovinos destinados a la producción de carne y leche; aunque en el trópico bajo, por sus propias caracteristicas se presentan ciertas adversidades climáticas y concomitantemente de suelos que de alguna manera interfieren con una buena nutrición de las pasturas. Esta situación, propia del trópico bajo, determina en gran medida, una disminución en la oferta natural de forrajes por una parte, y por otra, existe un deficiente suministro de minerales provenientes del suelo, lo que se traduce en una inadecuada alimentación animal, y en consecuencia, se presentan sistemas productivos deficientes (1).

Los minerales en la dieta animal aunque se requieran en cuantías mínimas, muchas veces no se les suministran en cantidades adecuadas y pueden ocasionar desórdenes metabólicos causando ineficiencia productiva en dichos animales. En los sistemas de producción bovina de carne (cría) se pretende conseguir cada día una mejor ganancia de peso diaria y por tanto los requerimientos nutricionales en general son cada vez más altos; sin desconocer que la energía y la proteína son factores primarios que se da por descontado su oferta en la dieta, pero su aporte se hace ineficiente si no se tiene en cuenta su interacción con los minerales y las vitaminas como nutrientes esenciales en la alimentación animal (2).

Como parte fundamental en un sistema de producción bovina la reproducción ejerce un rol muy importante en la productividad de cualquier empresa del sector. Un parto lo más cercano a 12-15 meses por hembra garantizaría una lactancia y al menos, una cría en un tiempo razonable para el auto reemplazo, lo que se traduciría en ingresos para el productor; de lo contrario se seguirá encontrando de cara con los bajos índices productivos, mayores número de días abiertos, intervalos entre partos incrementados, número de crías disminuidas y menor producción de leche para venta, derivado de la ineficiencia en el manejo de los minerales y en especial el cobre que cumple un papel fundamental en la reproducción de los bovinos. El objetivo de este manuscrito fue revisar algunos aspectos relacionados con el rol que tiene el cobre en la fisiología animal y en especial en la reproducción animal bovina.

En el país se ha hecho necesario para la competitividad y sostenibilidad económica de los diferentes sistemas de producción bovina que los animales expresen su máximo potencial productivo, lo que a su vez ha incrementado la demanda de sus requerimientos nutricionales. El contenido mineral que se encuentra en las pasturas es un factor limitante muy importante que está presenta en los sistemas de producción de bovinos.

Factores involucrados en los sistemas de producción (intrínsecos y extrínsecos) tales como el suelo, la fertilización, el uso de suplementos minerales de baja calidad, así como el incremento de los requerimientos minerales en los animales, probablemente determinen en las explotaciones ganaderas deficiencias crónicas de minerales. En estas condiciones, los animales presentan desde inadecuados índices productivos y reproductivos hasta la aparición de síntomas de enfermedades y en algunos casos alta mortalidad (1). Esta situación obliga a conocer de primera mano las necesidades de los minerales como el cobre $(\mathrm{Cu})$ en la producción bovina, sus características e implicaciones en la ganancia de peso, presentación de celos, así como las diferentes alternativas para el suministro de dicho mineral.

Función del cobre. El Cobre $(\mathrm{Cu})$ participa en varios procesos fisiológicos dentro de los cuales se encuentran la respiración celular, la formación de ósea, fisiología del miocardio, mielinización de la médula espinal, desarrollo del tejido conectivo, queratinización y pigmentación de algunos tejidos. En aquellas áreas en donde se presentan factores que interfieren con la absorción del $\mathrm{Cu}$, la mayor parte de las deficiencias están influidas por factores de la dieta (3). Por otra parte, el Cu también participa en la síntesis de hemoglobina, absorción 
y movilización del hierro $(\mathrm{Fe})$; es componente de varios sistemas enzimáticos que actúan, entre otros, en el sistema nervioso central y síntesis de melanina (4).

Alguna funciones del cobre en bovinos. El cobre cumple una función muy importante en la digestión de los forrajes, en la reproducción, en el sistema inmune y en el desarrollo óseo, muscular y dentario. De acuerdo con Febres (5), la ingesta deficiente de cobre y de vitaminas puede generar numerosas respuestas negativas en el animal, provocando disminución o reducción en algunas actividades tales como:

1. Consumo de forraje

2. Aprovechamiento de los alimentos

3. Ganancia de peso

4. Eficiencia reproductiva

5. Resistencia a las enfermedades

6. Peso al nacimiento

En los sistemas de producción bovina de carne, una de las deficiencias nutricionales de mayor impacto económico lo constituye la hipocruposis y afecta en mayor proporción a los animales jóvenes, en un rango de edad que va desde los tres y treinta y seis meses, pero afecta en mayor medida a los animales menores de los 12 meses.

Los animales que presentan deficiencias generarán pérdidas económicas porque tendrán una menor ganancia diaria de peso y adicionalmente podrán presentar dificultades o alteraciones osteoarticulares, afectando finalmente la tasa normal de crecimiento. Si la deficiencia ocurre en animales adultos, estos padecerán alteraciones en secreción hormonal, y por lo tanto, mostrarán perturbaciones en la duración el ciclo estral y en el celo (anestro), con el subsecuente impacto negativo en los índices reproductivos; en machos, se reduce la espermatogénesis (2).

Los signos clínicos de la deficiencia de cobre en general tienen que ver con trastornos bioquímicos que precedidos por disminuciones en las concentraciones hepáticas y plasmáticas de $\mathrm{Cu}$, generan una deficiente actividad enzimática, y en algunos casos más graves estarán ausentes algunas enzimas tisulares cobre dependientes (6).

Requerimientos. Los requerimientos del cobre varían de 4 a 15 ppm, rango que dependerá ostensiblemente de la concentración concomitante del molibdeno (Mo) y azufre (S). En la dieta rutinaria se recomienda una concentración de $10 \mathrm{ppm}$, con lo cual se mantendrían las concentraciones de $0.25 \%$ de azufre y de 2 mg de molibdeno, logrando así un equilibrio de conveniencia de acuerdo con lo comentado anteriormente. Es muy probable que dietas con menos de $10 \mathrm{ppm}$ de $\mathrm{Cu}$ cubran los requerimientos del hato; pero las dietas concentradas poseen usualmente mayor cantidad de Cu que los forrajes (7).

Es importante tener presente según Bauer et al (8), que los requerimientos de cobre son mayores cuando la concentración de los antagonistas naturales como el Mo, S y Fe se encuentra incrementada. Por otra parte, y no menos importante es saber que la absorción de Cu también se disminuye en presencia de ácido fítico y Zn, ácido ascórbico y Fe, así como en presencia de un pH alcalino. Por lo tanto es recomendable acompañar el suministro de $\mathrm{Cu}$ de aminoácidos, ya que estos favorecen su absorción.

Por otra parte, los animales jóvenes y en especial el hígado de los recién nacidos cuenta con reservas suficientes y además poseen una mayor capacidad de absorción que los adultos, pero en general los requerimientos de cobre podrían variar de acuerdo con las diferentes razas (8).

Deficiencia de cobre en bovinos. Cuando se ofrece una dieta sin cobre a bovinos, se pueden observar los siguientes trastornos generales: despigmentación con la formación de anteojeras blancas en animales de pelaje oscuro (acromotriquia), que corresponde a un signo temprano de la deficiencia; crecimiento lento; anestro (falta de celo o calor) y presentación de fracturas espontáneas en animales jóvenes (9). La deficiencia de cobre también se puede expresar en menor escala en la disminución de la producción de carne y leche; paralelamente pueden cursar la anemia y las ya comentadas anormalidades óseas. Es necesario tener muy en cuenta que en la alimentación de los bovinos deben estar presentes los minerales esenciales; ellos son muy importantes porque 
participan en complejos procesos metabólicos que tributan a la buena salud animal y producción animal. Cabe recordar que el cobre es uno de ellos, por lo que se debe tener muy presente (10).

La hipocupremia es frecuente en aquellos animales que consumen pastos con niveles inferiores a 4 ppm de $\mathrm{Cu}$ en materia seca (deficiencia primaria), así como en los casos en donde la concentración de alguno de sus antagonistas es alta (deficiencia secundaria). Generalmente esta situación se presenta cuando la concentración del cobre está por debajo de las 5 ppm y la del molibdeno es superior a las 3 ppm en el forraje que consumen; lo que correspondería a una relación de 2:1. También es muy probable que la deficiencia de $\mathrm{Cu}$ se presente cuando el ganado consume aguas muy duras, las cuales, por sus características contienen altas concentraciones de sulfatos (8).

De acuerdo con los requerimientos diarios de la dieta, a los minerales se clasifican en macro minerales (g/ día) y micro minerales $(\mathrm{mg} / \mathrm{día})$. Dentro de los macrominerales están el calcio, fósforo, magnesio, potasio, cloro, sodio y azufre, y dentro de los microminerales (oligoelementos) se clasifican el cobre, hierro, yodo, manganeso, cobalto, selenio y zinc.

Se conoce que la deficiencia de cobre en los bovinos tiene diversas sintomatologías, pero en general se puede manifestar por:

- Anestro

- Anormalidades óseas

- Desórdenes neurológicos

- Despigmentación del pelaje

- Respuesta inmunológica deficiente

- Anemia (la deficiencia de cobre limita la utilización de hierro)

Toxicidad del cobre. La concentración máxima que toleran los bovinos en la dieta es de 100 ppm, pero se debe tener en cuenta como se ha comentado anteriormente, que la concentración final del cobre en la dieta, estará afectada de la concentración del molibdeno y del azufre, así como del resto de los otros antagonistas del cobre que se encuentren en la dieta.

Los principales síntomas de toxicidad que presentan los animales son: mal estado general, debilidad, depresión, letargia, dolor abdominal con presencia de lo conocido vulgarmente como "vientre agalgado", sialorrea, trismo, bruxismo, ictericia y hemoglobinuria. Adicionalmente se presenta eritropenia, leucocitosis, neutrofilia, dolor abdominal, cólico, heces pastosas de color verdoso, marcha insegura, espasmos, parálisis; epistaxis y en ocasiones los animales pueden morir alrededor de las 48 horas (8).

Efectos del cobre sobre la reproducción. Aunque la infertilidad es un problema multifactorial, una considerable proporción de animales muestra una apropiada respuesta posterior a la suplementación mineral; pero es factible mejorar el comportamiento reproductivo de los bovinos afectados por hipocuprosis (11). La rentabilidad y la eficiencia en la ganadería bovina dependen de las capacidades reproductivas de los animales, la salud y el incremento de la productividad por área en explotación. Un parto lo más cercano al año por hembra incorporada a la reproducción, garantizaría una lactancia y concomitantemente una cría (12).

Se considera que la deficiencia de cobre $\mathrm{Cu}$ se constituye en uno de los déficits de minerales que más influye sobre el desempeño reproductivo, y algunos autores la clasifican como la segunda deficiencia más frecuente en bovinos manejados en pastoreo en el mundo y se ubica después de la de fósforo (12). Por otra parte, algunos autores sugieren que las carencias minerales están asociadas a diversos trastornos propios de la fertilidad en el ganado bovino criado en pastoreo. Se plantea que la pubertad tardía, involución uterina tardía, abortos, anestro, retención de placenta y hembras repetidoras de servicios, son eventos que están relacionados con hipocupremia (13). En la tabla 1 se observa el efecto de la administración de una solución de sulfato de cobre $(25 \mathrm{mg} / \mathrm{ml})$ vía parenteral sobre el desempeño reproductivo en hembras bovinas en pastoreo con presentación de celos espontáneos. 
TABLA 1

Efecto de la administración de una solución de sulfato de cobre $(25 \mathrm{mg} / \mathrm{ml})$ vía parenteral sobre el comportamiento reproductivo en hembras bovinas en pastoreo con celo normal

\begin{tabular}{|l|c|c|}
\hline \multicolumn{1}{|c|}{ INDICADORES } & GRUPO CONTROL & GRUPO TRATADO \\
\hline Número de animales & 60 & 60 \\
\hline Condición corporal media & 3.6 & 3.9 \\
\hline Valores del cobre sérico (mmol /l) & $11.04 \pm 1.08^{\mathrm{a}}$ & $13.39 \pm 2.37^{\mathrm{b}}$ \\
\hline Hembras que presentaron celo & 32 & 57 \\
\hline Hembras gestantes & 22 & 51 \\
\hline Concepción (\%) contra las hembras que presentaron celo & $68.7^{\mathrm{a}}$ & $89.5^{\mathrm{b}}$ \\
\hline Concepción (\%) contra el total de hembras del grupo & $36^{\mathrm{a}}$ & $85^{\mathrm{b}}$ \\
\hline Beneficio económico (US\$ por novillas) & 1.05 & 2.5 \\
\hline $\begin{array}{l}\text { ab } \\
\text { (13). medias con diferentes letras en la misma fila difieren significativamente para p<0.05. Fuente }\end{array}$ \\
\hline
\end{tabular}

Como se mencionó anteriormente, la deficiencia de $\mathrm{Cu}$ puede ser de origen primario o secundario; pero también se describe una forma terciaria, la que involucra falla en la actividad de metaloenzimas del cobre, pero la deficiencia secundaria es la más frecuente (14).

Con relación al mecanismo implicado con los trastornos reproductivos, el zinc, cobre y manganeso desempeñan un papel esencial en la reproducción. Por ejemplo, las bajas concentraciones de zinc y cobre durante la maduración in vitro induce una apoptosis, lo cual afecta la integridad del ADN de las células del cumulus oophorus, lo que a su vez afecta el normal desarrollo de los oocitos bovinos. La deficiencia de cobre afecta la reproducción debido a que este participa en la reducción del estrés oxidativo a nivel ovárico, así como en el mantenimiento de la secreción de gonadotropinas (14).

Trastornos en la reproducción bovina por hipocruposis. Se ha informado disminución de la concepción, ciclos anovulatorios y anestros, disminución del pico de $\mathrm{LH}$ y una reducción $(\mathrm{p}<0.05)$ en los números y la frecuencia de pulsos de $\mathrm{LH}$ en ganado con deficiencia de $\mathrm{Cu}$ al compararlo con animales normocuprémicos. Se ha informado que el $\mathrm{Cu}$ está relacionado con la síntesis y secreción de las gonadotropinas, modulando la capacidad de liberación de la hormona luteinizante $(\mathrm{LH})$, por lo cual se sugiere que la subfertilidad e infertilidad se deban a inhibición en la síntesis de hormonas gonadotrópicas, así como la reducción del estrés oxidativo a nivel ovárico cuando se presenta hipocruposis. En los rumiantes, el folículo dominante es la principal fuente (96\%) de la secreción del estradiol del ovario (síntomas externos de celo) y además, es el responsable de liberar el pico de estradiol del folículo preovulatorio indispensable para que ocurra la ovulación luego de la estimulación y liberación del pico de LH. Por consiguiente, la interferencia en el desarrollo normal de este folículo podría perturbar el estro y la ovulación. La deficiencia de Cu además de tener efectos directos sobre la producción hormonal, integridad del tracto reproductor y función ovárica, también está implicado en la muerte embrionaria y la repetición de servicio (12)

Métodos de suplementación del cobre. Los minerales son necesarios para transformar la proteína y la energía de la dieta en componentes del organismo o en productos animales. También contribuyen con el organismo animal a combatir las enfermedades, manteniendo al animal en buen estado de salud. Algunos autores han considerado que los minerales constituyen el tercer grupo limitante en la nutrición animal con mayor potencial de utilizar dado el menor costo para incrementar la producción del ganado (15).

Cumplir con los requisitos minerales para el ganado en pastoreo a menudo se convierte en un desafío. Identificar los minerales que no son suministrados en cantidades adecuadas por la dieta es necesario. Para agravar este esfuerzo se encuentran las diversas interacciones entre minerales y los desequilibrios que ocurren entre ellos. Por lo tanto, es necesario tomar una buena decisión sobre el método de suplementación mineral que se vaya a suplementar. Por un lado se utiliza la fertilización del suelo como un método indirecto y 
aplicaciones foliares pero estas pueden alterar el pH del suelo. Sin embargo, la fertilización mineral solo debe practicarse para mejorar las características de producción del forraje. La fertilización mineral no es un método eficiente para aumentar la ingesta de minerales a los animales (16).

Dentro de los métodos directos de suplementación mineral se encuentran los siguientes:

- Adicionar minerales en el agua

- Inmersión oral

- Aplicación parenteral

- Bolos ruminales

- Suplementación mineral

Los más frecuentemente utilizados son los de uso parenteral y la suplementación mineral. La suplementación mineral debe adecuarse a las diferentes condiciones del terreno y suelo de cada finca, esto con el fin de determinar una mayor eficacia en la suplementación y garantizar el consumo adecuado de minerales (16). En condiciones de trópico bajo, Patiño et al (17) destacan que el pastoreo es la principal estrategia que se utiliza para la alimentación de los rumiantes, lo que de alguna manera agudiza la ingestión de minerales pues en realizad no se logra conocer del todo la ingestión de los mismos ni la composición mineral de los forrajes. Cementan ellos que existe información sobre las exigencias minerales en rumiantes, pero que no es nada fácil dilucidar la interrelación de la mayor parte de modelos para predecir la exigencia mineral derivada de la consulta de una sola fuente de información.

Formular suplementos minerales de libre elección puede volverse complejo debido a las diferencias en los minerales encontrados en cada forraje y a su biodisponibilidad, las interacciones entre minerales y la dificultad en la evaluación del estado mineral de los animales. La suplementación se debe realizar bajo los parámetros de un análisis bromatológico con el fin de conocer las deficiencias y excesos de macro y microminerales $(15,18)$.

La práctica más utilizada para suplementar el cobre es a través de soluciones inyectables de compuestos orgánicos de cobre tales como: glicinatos, edeatos o lactatos cada tres o cuatro meses (10), lo que permite incrementar la disponibilidad a nivel tisular, evitando las interacciones en el tracto gastrointestinal (18).

En la tabla 2 se muestran los resultados de un experimento hecho en terneros con pesos entre 180 y $210 \mathrm{~kg}$. Se conformaron dos grupos de 25 animales cada uno. El grupo A se tomó como control y el B fue inyectado vía subcutánea con $50 \mathrm{mg}$ de $\mathrm{Cu}$; la primera aplicación se realizó al inicio del experimento, repitiéndose cada 60 días hasta completar tres aplicaciones (19). Los resultados fueron los siguientes:

TABLA 2

Comparación (g) entre grupos de tratamientos en diferentes tiempos de muestreo

\begin{tabular}{|c|c|c|}
\hline Días & Testigo & Tratamiento \\
\hline 30 & $373.33+117^{a}$ & $408+54.67^{\mathrm{a} *}$ \\
\hline 60 & $442.66+64.92^{b}$ & $516+66.05^{2 *}$ \\
\hline 90 & $862.66+136.53^{b}$ & $938.66+72.44^{2 *}$ \\
\hline 120 & $848.66+104.5^{b}$ & $1006.6+103.63^{a x}$ \\
\hline 150 & $852+117.8^{\mathrm{b}}$ & $1072+98.92^{\text {a* }}$ \\
\hline 180 & $954.66+193.73^{b}$ & $1141.3+111.08^{a x}$ \\
\hline Total & $722.2+72.2^{b}$ & $847.1+57.4^{\mathrm{a} * *}$ \\
\hline \multicolumn{3}{|c|}{$\begin{array}{l}\text { ab diferentes letras en la misma fila indican diferencias } \\
\text { estadisticas significativas (t Student para muestras } \\
\text { independientes) } p<0.01 \text {. * } p<0.001 \text {. Fuente: (19). }\end{array}$} \\
\hline
\end{tabular}

En la tabla 3, se presenta el trabajo realizado por García-Díaz et al (20) en donde se muestra el efecto de la suplementación parenteral de cobre sobre la ganancia en terneros de ceba. 
TABLA 3

Distribución de los tres tratamientos realizados al azar en grupos de 15 terneros

\begin{tabular}{|c|c|c|c|}
\hline Grupos & Primera dosis Día 0 & Segunda dosis Día 60 & Tercera dosis Día 120 \\
\hline 1 & Placebo & Placebo & Placebo \\
\hline 2 & $25 \mathrm{mg} \mathrm{de} \mathrm{Cu}$ & $40 \mathrm{mg}$ de $\mathrm{Cu}$ & $40 \mathrm{mg}$ de $\mathrm{Cu}$ \\
\hline \multirow{3}{*}{3} & $25 \mathrm{mg}$ de $\mathrm{Cu}$ & $40 \mathrm{mg}$ de $\mathrm{Cu}$ & $40 \mathrm{mg}$ de $\mathrm{Cu}$ \\
\hline & $50 \mathrm{mg}$ de $\mathrm{Zn}$ & $80 \mathrm{mg}$ de $\mathrm{Zn}$ & $80 \mathrm{mg}$ de $\mathrm{Zn}$ \\
\hline & $25 \mathrm{mg}$ de $\mathrm{Mn}$ & $40 \mathrm{mg}$ de $\mathrm{Mn}$ & $40 \mathrm{mg}$ de $\mathrm{Mn}$ \\
\hline
\end{tabular}

La ganancia de peso diaria de peso en cada grupo (Tabla 4), mostró que a los 60 días fueron diferentes para los tres grupos y fue superiores $(p<0.05)$ en el grupo 2 . Hubo diferencias significativas $(p<0.05)$ a favor de los grupos 2 y 3 a los 120 días y $(\mathrm{p}<0.001)$ a los 180 días. Estos resultados indicaron que los suplementos empleados tuvieron un efecto favorable sobre este indicador (20).

TABLA 4

Comparación del peso de los terneros (kg) y GMD (g) entre los grupos de tratamiento en diferentes momentos del muestreo.

\begin{tabular}{|c|c|c|c|c|c|c|}
\hline \multirow{3}{*}{ Días } & \multicolumn{6}{|c|}{ Grupos } \\
\hline & \multicolumn{2}{|c|}{1} & \multicolumn{2}{|r|}{2} & \multicolumn{2}{|c|}{3} \\
\hline & Peso & GMD(g) & Peso & GMD (g) & Peso & GMD(g) \\
\hline 60 & $68.53 \pm 2.82^{b}$ & $282.22 \pm 26.32^{c}$ & $75.53 \pm 3.18^{2}$ & $426.66 \pm 52.6^{2}$ & $72.8 \pm 4.31^{b_{*}}$ & $377.8 \pm 71.17^{\mathrm{b}_{*}}$ \\
\hline 120 & $87.33 \pm 3.79$ & $313.33 \pm 26.8^{\mathrm{b}}$ & $97.53 \pm 3.81^{\text {2 }}$ & $366.7 \pm 35.07^{2}$ & $93.66 \pm 4.18^{b_{*}}$ & $347.77 \pm 27.36^{\text {a* }}$ \\
\hline 180 & $106 \pm 3.29^{b}$ & $311.11 \pm 22.41^{b}$ & $119.13 \pm 4.67^{a}$ & $360 \pm 61.8^{\mathrm{a}}$ & $118.93 \pm 3.43^{2 * * *}$ & $371.1 \pm 62.72^{\text {a**** }}$ \\
\hline
\end{tabular}

Cabe resaltar que los resultados de estos estudios pueden variar entre los mismos y con muchos otros realizados según sea el caso, ya que hay que tener en cuenta todo tipo de variables, animales utilizados, edad al tratamiento, sexo, alimentación, manejo, tipo de suelo, etc.

La forma indirecta de administración mineral debe considerar el manejo apropiado del suelo, adiciones de compuestos que favorezcan el incremento del $\mathrm{pH}$ y se mejore biodisponibilidad de ciertos minerales en la planta. La captación de los minerales del suelo por parte de las plantas es diferente, por lo que se recomienda la utilización de diferentes especies de forrajes que se desarrollen y produzcan en el mismo suelo; de esta manera se dispondrá de una variada concentración de algunos minerales (5). De otro lado, hay que tener en cuenta que a medida que las plantas maduran, el contenido mineral disminuye debido a un proceso natural de dilución y traslado de nutrientes a la raíz (3).

Para comprender los diferentes resultados obtenidos por distintos investigadores incluye: región geográfica, material madre, topografía, textura de los suelos, composición botánica de las praderas, variación estacional, disponibilidad de materia seca, fertilización y época de año. Por otra parte, la relación entre los requerimientos de un nutriente y su contenido en la pastura es solamente la primera aproximación al status nutricional de animales en pastoreo, dado que el contenido en la ingesta puede ser diferente debido al fenómeno de selectividad que es intrínseco de los animales. Igualmente se deberá considerar la biodisponibilidad del elemento en cuestión y la capacidad de reserva en el organismo del mismo (3). Por lo tanto, en cada finca se debe determinar la calidad del suelo, de sus pasturas y la misma interacción de los macro y microminerales para saber la acción correcta de los minerales en el organismo del animal según el tipo de suelo.

En las áreas en donde los forrajes tienen un alto contenido de molibdeno, es recomendable triplicar o cuadruplicar la concentración del cobre. Es tan crítica la situación que algunos autores consideran que 3 ppm de molibdeno, o $0.5 \%$ de azufre pueden reducir hasta el $50 \%$ la utilización del cobre. Esta situación indica que 
la concentración de cobre para contrarrestar los efectos del molibdeno y el azufre, se torna en un problema complejo que debe ser abordado de acuerdo con la caracteristicas de cada área (21). Finalmente no hay que olvidar que una baja eficiencia reproductiva induce una pérdida económica porque se incrementan los costos de producción ya que se incrementa el intervalo entre partos y por ende la natalidad con las consecuencias de producir menos terneros y menos leche en los periodos productivos de los animales (22).

En conclusión, en cada región se podrá encontrar una variedad de suelos que se deberán examinar para conocer que desequilibrios minerales se encuentran en él y por lo tanto en sus pasturas. Su disponibilidad también estará restringida por mal uso de los suelos, forrajes y abuso de herbicidas. Por lo general el desconocimiento de la importancia de los macro y micro minerales en la producción han llevado a una inadecuada dieta para los animales, y por lo tanto su ingesta dependerá de su disponibilidad en los forrajes.

Un correcto balance mineral en la dieta animal, así como un adecuado consumo, supone una mejora en las tasas de preñez, tasa de natalidad, aumento de la producción en leche, mejores pesos al destete y ganancia de peso. Asimismo, se lograría alcanzar una mejor rentabilidad de los sistemas de producción bovina.

Como los forrajes no aportan la suficiente concentración de cobre para mantener parámetros óptimos en la vida del animal, se deben trazar estrategias para asegurar el suministro del mismo; bien sea mediante sales bien balanceadas o parenteralmente cuando sea necesario o trazar un plan estratégico de acuerdo con el sistema de producción que se vaya a intervenir.

\section{REFERENCIAS}

1. Garmendia J. Los minerales en la Reproducción Bovina. Maracay, Venezuela: Universidad Central de Venezuela, Facultad de Ciencias Veterinarias; 2006.

2. Repetto J, Donovan A, García F. Carencias minerales, limitantes de la producción. Sitio argentino de Producción Animal [Internet], [02 octubre 2017]. 2004. URL Valida en: http://www.produccion-animal.com.ar/ suplementacion_mineral/18-carencias_limitantes_produccion.pdf

3. Pittaluga O. Rol de los minerales en la producción de bovinos para carne en Uruguay. Unidad de Comunicación y Transferencia de Tecnología de INIA: Montevideo, Uruguay; 2009. http://www.ainfo.inia.uy/digital/ bitstream/item/2794/1/18429021009100908.pdf

4. Stahringer R, Mcdowell L. Suplementación con cobre inyectable en terneros y vacas con hipocupremia. INTA: Argentina; 2012; 154:272-280. https://inta.gob.ar/documentos/suplementacion-con-cobre-inyectableen-terneros-y-vacas-con-hipocupremia

5. Febres A. La nutrición mineral del ganado vacuno. Universidad de Zulia: Venezuela; 2008.

6. Greene L. Designing mineral supplementation of forage programs for beef cattle. Journal of Animal Science 2000; 77(Suppl):1-9. DOI: https://doi.org/10.2527/jas2000.00218812007700ES0046x

7. Rosa D, Mattioli G. Metabolismo y deficiencia de cobre en los bovinos. Analecta Veterinaria. 2002; 22(1):1-7. URL Valida en: http://www.fcv.unlp.edu.ar/images/stories/analecta/ vol_22_n1/051_VE22n1_rosa_metabolismo_cobre.pdf

8. Bauer D, Rush I, Rasby R. Minerales y vitaminas en bovinos de carne. Sitio Argentino de Producción Animal; 2009. URL Disponible en: http://www.produccion-animal.com.ar/suplementacion_mineral/118minerales_vitaminas-Nebraska.pdf

9. Villanueva G. Nutrición del Ganado. Sitio Argentino de Producción Animal; 2011. URL Disponible en: http:// www.produccion-animal.com.ar/suplementacion_mineral/145-cobre.pdf

10. Mufarrege D. El cobre en la Ganadería Del Nea. [Internet]. [cited 5 November 2017]. Sitio Argentino de Producción Animal; 2003; Available from: http://www.produccion-animal.com.ar/ suplementacion_mineral/49-cobre_en_el_nea.pdf

11. Pedrosa R, Gonzalez N, Roller F. Efecto de la suplementación con cobre en la reproducción de la hembra bovina en pastoreo. La Técnica 2014; 13:26-31. 
12. Ramon J. Relación entre la cupremia y los indicadores reproductivos de la hembra bovina. [Tesis]. La Habana: Universidad Agraria De La Habana; 2008.

13. Garcia J, Cuesta M, Pedroso R, Gutierrez M, Mollineda A, Figueredo J. Efecto del cobre sobre la reproducción en novillas lecheras de Cuba. Rev MVZ Córdoba 2006; 11(2):790-798. https://doi.org/10.21897/rmvz.442

14. Hernández W, Ocampo P, Montoya L, Bustamante J. Suplementación parenteral de cobre y su relación con índices de fertilidad en bovinos de regiones con altos niveles de molibdeno en el Magdalena Medio colombiano. Revista Veterinaria y Zootecnia. 2016; 10(1):01-12. DOI: https://doi.org/10.17151/vetzo.2016.10.1.1

15. Castelli M, Abdala A, Ibarlucea J, Diruscio I, Cuatrin A. Deficiencia de cobre en bovinos: Algunas preguntas y respuestas [Internet]. Todoagro. [citado 7 Noviembre 2017]. 2011. Disponible en: http:// www.todoagro.com.ar/noticias/nota.asp?nid=16627

16. Bejarano R, Forero N. Efecto de la suplementación de zinc, cobre quelatados y selenio de fuente orgánica sobre los niveles sanguíneos y la calidad de la leche en la raza Holstein en el cic santa María. Colombia: Universidad De La Salle; 2007.

17. Patiño R, Da Silva Filho J Perez P. Modelos de predicción de exigencias minerales para rumiantes. Revista Colombiana De Ciencia Animal - RECIA, 2011:3(2),344-365 https://doi.org/10.24188/recia.v3.n2.2011.409

18. Salamanca A. Suplementación de minerales en la producción bovina. REDVET. 2010; 11(9). http:// www.veterinaria.org/revistas/redvet/n090910/091009.pdf

19. Mora R, Herrera A, Sanchez D, Chicco C, Godoy S, DePablos L. Suplementación parenteral con cobre y zinc en vacunos machos mestizos Brahman en los Llanos Occidentales de Venezuela. Rev Fac Agron (UCV) 2010; 36(3):2-10. http://saber.ucv.ve/ojs/index.php/rev_agro/article/view/10838

20. García-Díaz J, Noval-Artiles E, Pérez-Bello A, Hernández-Barreto M, Pérez-González Y. Efecto de la suplementación parenteral del cobre sobre la ganancia de peso de toros en ceba. Rev MVZ Córdoba 2010; 22(2):5821. https://doi.org/10.21897/rmvz.1009

21. Noval E, García J, García R, Quiñones R, Mollineda Á, Munyori H. Evaluación del efecto de la suplementación parenteral de cobre y un complejo de cobre $(\mathrm{Cu})$, zinc $(\mathrm{Zn})$ y manganeso $(\mathrm{Mn})$ sobre la hemoquímica y la ganancia de peso en terneros. RedVet. 2012; 13(10):3-10.

22. Granja SY, Cerquera GJ, Fernandez BO. Factores nutricionales que interfieren en el desempeño reproductivo de la hembra bovina. Revista Colombiana De Ciencia Animal - RECIA. 2012; 4(2):458-472. https:// doi.org/10.24188/recia.v4.n2.2012.227

Los autores permiten a RECIA reimprimir el material publicado en él. En caso de que un autor quiera traducir o usar una publicación parcial o completa de nuestro Diario, el autor debe obtener un permiso por escrito del editor de la revista.

CC BY 